\title{
Effect of chest wall vibration on the canine diaphragm during breathing
}

\author{
D. Leduc*, A. De Troyer ${ }^{\#}$
}

Effect of chest wall vibration on the canine diaphragm during breathing. D. Leduc, A. De Troyer. C) ERS Journals Ltd 2002.

ABSTRACT: High-frequency mechanical vibration of the ribcage reduces dyspnoea in patients with chronic obstructive pulmonary disease, and the suggestion has been made that this effect might be related to a decrease in central respiratory drive resulting from an increase in afferent inputs from intercostal muscles. In the present studies, the effects of ribcage vibration on central respiratory drive have been assessed without the confounding influence of conscious reactions.

The electromyographic (EMG) activity of the diaphragm and the changes in pleural $(P$ pl) and abdominal $(P$ ab) pressure were measured in six anaesthetized, spontaneouslybreathing dogs while the rostral, the middle, or the caudal portion of the ribcage was vibrated at intervals during inspiration. The EMG activity of the external and parasternal intercostals was also measured.

Ribcage vibration consistently elicited a marked increase in the inspiratory EMG activity recorded from the external intercostals, thus indicating that the procedure did activate intercostal muscle spindles. However, no alteration in diaphragmatic or parasternal intercostal EMG activity was seen in any animal. Transdiaphragmatic pressure and the relationship between $\Delta P$ ab and $\Delta P$ pl during inspiration were also unaltered.

The authors conclude that ribcage vibration and, with it, stimulation of external intercostal muscle spindles has no significant influence on phrenic motoneurones or on medullary inspiratory neurones. It is unlikely, therefore, that the beneficial effect of the procedure on dyspnoea results from a specific reduction in central respiratory drive. Eur Respir J 2002; 19: 429-433.
*Intensive Care Unit, Saint-Pierre University Hospital, Brussels and ${ }^{\#}$ Laboratory of Cardiorespiratory Physiology, Brussels School of Medicine, and Chest Service, Erasme University Hospital, Brussels, Belgium.

Correspondence: A. De Troyer Chest Service

Erasme University Hospital

Route de Lennik, 808

1070 Brussels

Belgium

Fax: 3225554411

Keywords: Control of breathing dyspnoea

respiratory muscles

Received: May 52001

Accepted after revision October 10 2001

This work was supported, in part, by a research grant from the Brussels School of Medicine.
Although medical treatment of airflow obstruction has made significant progress in the last $20 \mathrm{yrs}$, dyspnoea remains a major cause of disability in many patients with chronic obstructive pulmonary disease (COPD). Recent studies, however, have shown that this symptom could be significantly relieved by the application of high-frequency mechanical vibration to the parasternal region of the ribcage during inspiration [1, 2]. Vibration of the parasternal region has also been shown to reduce the sense of effort in healthy individuals breathing carbon dioxide $\left(\mathrm{CO}_{2}\right)$ enriched gas mixtures or breathing against inspiratory resistive loads $[3,4]$. The mechanism of this beneficial effect is uncertain, but high-frequency vibration is known to be a potent stimulus of spindle primary endings in limb muscles [5, 6]. In addition, in agreement with earlier animal experiments by REMMERs [7, 8], Homma et al. [9] have reported a decrease in diaphragmatic and parasternal intercostal inspiratory electromyographic (EMG) activity during vibration of the lower intercostal spaces in normal subjects. To the extent that medullary inspiratory neurone activity appears to be one of the determinants of dyspnoea [10-12], the suggestion has therefore been made that the beneficial effect of ribcage vibration might be, in part, related to a reduction in central respiratory drive induced by increased afferent inputs from intercostal muscles $[1,3,4]$.

Homma et al. [9], however, have reported also that vibration of the upper portion of the human ribcage during inspiration results in an increased parasternal intercostal inspiratory activity, and that vibration of the lower portion of the ribcage induces an increased activity in the caudal internal intercostal muscles. Conversely, recent studies in dogs have shown that when the upper ribcage is vibrated during inspiration, the inspiratory activity recorded from the external intercostal muscles in the rostral interspaces is consistently and markedly increased, but the activity recorded from the parasternal intercostals is unaltered [13]. This greater sensitivity to vibration of the external intercostals is fully consistent with their much greater density in muscle spindles [14]. Moreover, internal intercostal activity remained unchanged during vibration of the lower portion of the ribcage [15], and this led to the conclusion that the alterations in respiratory muscle activity reported in humans [9] were probably the result of conscious reactions to the perception of vibration in the ribcage or the abdomen, rather than reflex specific changes in central respiratory drive $[13,15]$. In the present studies, the validity of this conclusion has been tested further by examining 
the effects of ribcage vibration on the canine diaphragm; since the animals were anaesthetized, all potential conscious reactions to vibration were eliminated.

\section{Methods}

The experiments were carried out on six adult mongrel dogs $(14-20 \mathrm{~kg})$, anaesthetized with pentobarbital sodium (initial dose, $25 \mathrm{mg} \cdot \mathrm{kg}^{-1}$ intravenously). The animals were placed in the supine posture and intubated with a cuffed endotracheal tube, and a venous cannula was inserted in the forelimb to give maintainance doses of anaesthetic. A catheter was also inserted in the femoral artery to monitor blood pressure and sample arterial blood periodically for blood gas analysis. The ribcage and intercostal muscles were exposed on the right side of the chest from the first through the eleventh rib by deflection of the skin and underlying muscle layers, and pairs of silver hook electrodes spaced 3-4 mm apart were implanted in the parasternal and external intercostal muscles of the third interspace. Each electrode pair was implanted in parallel fibres in the portion of the muscle receiving the greatest inspiratory neural drive; implantation of the parasternal electrodes, therefore, was made in the muscle bundles near the sternum [16], and implantation of the external intercostal electrodes was made in the dorsal portion of the muscle immediately ventral to the angle of the ribs [17, 18]. The abdomen was then opened by a midline incision from the xiphisternum to the umbilicus, and an additional pair of hook electrodes was implanted in the costal portion of the right hemidiaphragm, after which the abdominal wall was carefully sutured in two layers.

\section{Measurements}

The animals were allowed to recover for $30 \mathrm{~min}$ after instrumentation, after which measurements of EMG activity, airflow, lung volume, and pleural and abdominal pressure were made. The three EMG signals were processed with amplifiers (model 830/1; CWE Inc., Ardmore, PA, USA), bandpass filtered below 100 and above $2,000 \mathrm{~Hz}$, and rectified before their passage through leaky integrators with a time constant of $0.2 \mathrm{~s}$. Airflow was measured at the endotracheal tube with a heated Fleisch pneumotachograph connected to a differential pressure transducer (Validyne, Northridge, CA, USA), volume was obtained by electronic integration of the flow signal, and pleural and abdominal pressures were measured with balloon-catheter systems placed in the middle third of the oesophagus and the abdominal cavity, respectively. The oesophageal balloon contained $0.5 \mathrm{~mL}$ of air, and the abdominal balloon was filled with $1.0 \mathrm{~mL}$ of air.

\section{Protocol}

The measurements were made while the animal was spontaneously breathing room air. The parasternal
EMG signal was continuously monitored on a loudspeaker to provide the investigator with a precise phase reference, and every 5-10 breaths, vibrations were applied during inspiration to the parasternal intercostal muscle in the third interspace, immediately medial to the costochondral junction. As in previous studies [13, 15], these vibrations were delivered using a commercially available vibrator (model V. 101; LDS, Royston, UK) which was adjusted so that the amplitude of its moving element and the frequency of vibration were $2.0-2.5 \mathrm{~mm}$ and $40 \mathrm{~Hz}$, respectively. The vibrator was held manually throughout, perpendicular to the muscle, so that the site and the force of application could be well controlled; the area of contact between the vibrator and the muscle was approximately $1.8 \mathrm{~cm}^{2}$. In each animal, at least 10 breaths with in-phase vibration were obtained.

When this procedure was completed, the two pairs of EMG electrodes implanted in the third interspace were transferred to the parasternal and external intercostal muscles in the sixth interspace, and in-phase vibration was applied at intervals to the sixth parasternal intercostal. The external intercostal electrodes were finally transferred to the ninth interspace, and in-phase vibration was delivered either to the ventral or the lateral portion of the ninth and tenth interspaces. As for the third interspace, at least 10 breaths with in-phase vibration were obtained for each interspace in every animal.

The animals were maintained under light surgical anaesthesia throughout the measurements. Supplementary doses of anaesthetic $\left(1-2 \mathrm{mg} \cdot \mathrm{kg}^{-1}\right)$ were given at regular intervals to ensure that there was no spontaneous movement of the fore- or hindlimbs, no flexor withdrawal of the forelimb, and no pupillary light reflex; the corneal reflex, however, was kept present. Rectal temperature was also maintained constant between $36-38^{\circ} \mathrm{C}$ with infrared lamps. At the end of the experiment, the animal was given an overdose of anaesthetic (30-40 $\mathrm{mg} \cdot \mathrm{kg}^{-1}$ intravenously).

\section{Data analysis}

Phasic inspiratory EMG activity in the diaphragm, parasternal intercostal, and external intercostal during each vibrated breath was compared with the activity recorded during the immediately preceding nonvibrated (control) breathe, by measuring the peak height of the integrated EMG signal in arbitrary units. The duration of inspiration (inspiratory time, $t \mathrm{I}$ ), defined as the period beginning at the onset of the diaphragmatic inspiratory burst and concluding with the peak diaphragmatic activity, did not show any consistent alteration with vibration in any animal (see Results). The changes in peak activity during vibration were therefore independent of the changes in neural inspiratory time and chemical respiratory drive that might have occurred. One study animal, however, did not have any external intercostal activity in the sixth or third interspace in the control breaths, even though the electrodes were inserted in the most dorsal portion of the muscle. Consequently, to allow comparison between the three muscles in 
the different animals, the inspiratory EMG activity recorded during each control breath was expressed as a percentage of the activity recorded during the subsequent vibrated breath.

Transdiaphragmatic pressure $(P \mathrm{di})$ in these breaths was determined by subtracting the change in pleural pressure $(P \mathrm{pl})$ at peak inspiration from the change in abdominal pressure $(P \mathrm{ab})$. Measurements of EMG activity and pressure were averaged over all trials in each individual animal, and they were then averaged for the animal group. Statistical assessments of the effects of in-phase vibration on inspiratory EMG activity, $P$ di, tidal volume, and $t$ I were made by using paired t-tests; the criterion for significance was taken as $\mathrm{p}<0.05$.

\section{Results}

The animals had a mean \pm SEM carbon dioxide tension in arterial blood $\left(P \mathrm{a}, \mathrm{CO}_{2}\right)$ of $38.5 \pm 1.6 \mathrm{mmHg}$ and a mean oxygen tension in arterial blood $\left(\mathrm{Pa}, \mathrm{O}_{2}\right)$ of $90.6 \pm 3.8 \mathrm{mmHg}$, and the effects of in-phase vibration of the third interspace on parasternal intercostal, external intercostal and diaphragmatic EMG activity are illustrated by the records of a representative animal in figure 1. Phasic inspiratory activity in the external intercostal increased with each vibration in every animal. However, no animal had any consistent increase or decrease in diaphragmatic EMG activity. For the animal group, therefore, peak diaphragmatic activity in the control breaths averaged $99.3 \pm 0.9 \%$ of the activity in the vibrated breaths (nonsignificant (NS)) whereas peak external intercostal activity in the control breaths was only $23.4 \pm 11.4 \% \quad(\mathrm{p}=0.001)$. Parasternal intercostal activity in the control breaths was also $102.1 \pm 3.3 \%$ of the activity in the vibrated
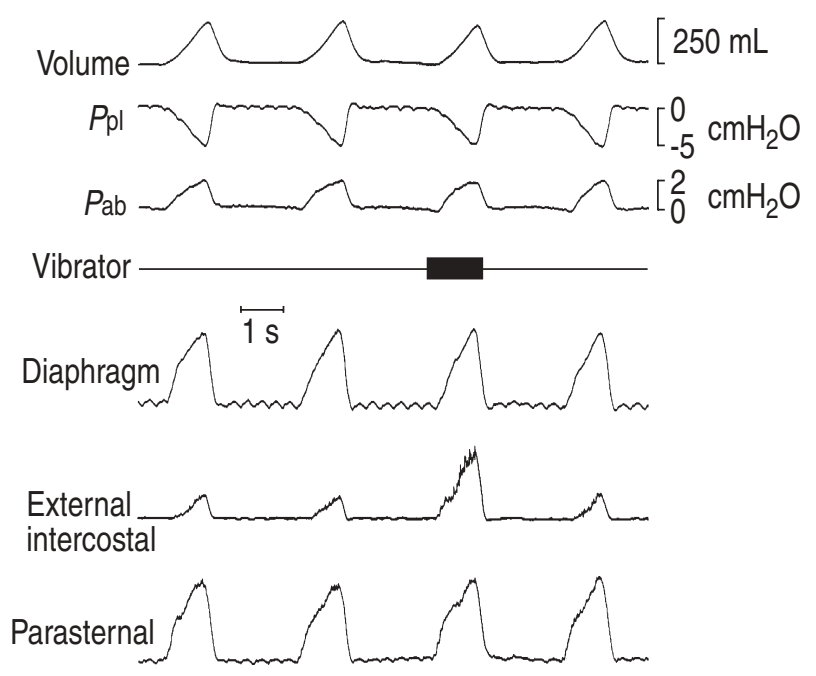

Fig. 1.-Response of the diaphragm, external intercostal, and parasternal intercostal muscles (third interspace) to in-phase vibration of the third interspace in a representative animal. Note the marked increase in external intercostal inspiratory activity during vibration. Diaphragmatic and parasternal intercostal activities, however, were unchanged. $P$ pl: pleural pressure; $P$ ab: abdominal pressure.

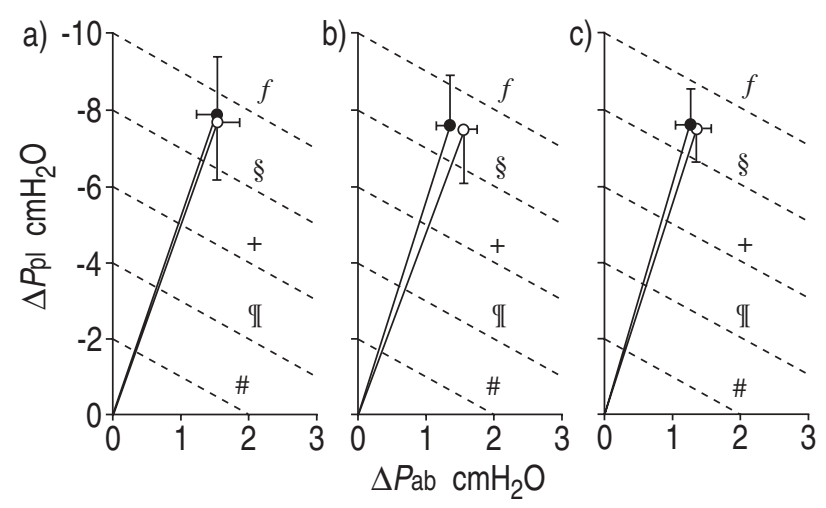

Fig. 2. - Effect of in-phase vibration of the a) third, b) sixth, and c) ninth interspace on the relationship between $\Delta$ abdominal pressure $(P \mathrm{ab})$ and $\Delta$ pleural pressure $\left(P_{\mathrm{pl}}\right)$ during inspiration. Average data of six animals; bars \pm SEM. Whatever the site of application, vibration did not alter the $P \mathrm{ab}-P_{\mathrm{pl}}$ relationship. Closed circles correspond to the control, nonvibrated breaths, and open circles correspond to the vibrated breaths. Dashed lines, isopleths corresponding to a transdiaphragmatic pressure of ${ }^{\#}: 2$; ๆ: 4; ${ }^{+}: 6 ;{ }^{\S}: 8 ;$ and $^{f}: 10 \mathrm{cmH}_{2} \mathrm{O}$.

breaths (NS). Pdi remained also unchanged (control: 9.4 $\pm 1.9 \mathrm{cmH}_{2} \mathrm{O}$; vibration: $9.3 \pm 1.8 \mathrm{cmH}_{2} \mathrm{O}$; NS), as did the relationship between $\Delta P$ ab and $\Delta P$ pl (fig. $2 \mathrm{a}$ ). $t$ I (control: $0.91 \pm 0.12 \mathrm{~s}$; vibration: $0.91 \pm 0.12 \mathrm{~s}$; NS) and tidal volume (control: $303 \pm 52 \mathrm{~mL}$; vibration: $295 \pm 52 \mathrm{~mL}$; Ns) were unaltered as well.

In-phase vibration of the sixth interspace had essentially similar effects. Peak external intercostal inspiratory activity in the control breaths was $51.1 \pm$ $12.8 \%$ of the peak activity recorded in the vibrated breaths $(\mathrm{p}=0.01)$, but diaphragmatic activity during control averaged $101.4 \pm 1.0 \%$ of the activity during vibration (NS). Parasternal intercostal activity, $t \mathrm{I}$ (control: $0.96 \pm 0.13 \mathrm{~s}$; vibration: $0.99 \pm 0.15 \mathrm{~s}$; Ns), tidal volume (control: $288 \pm 51 \mathrm{~mL}$; vibration: $286 \pm 53 \mathrm{~mL}$; NS), $P$ di (control: $9.0 \pm 1.5 \mathrm{cmH}_{2} \mathrm{O}$; vibration: $9.1 \pm$ $1.6 \mathrm{cmH}_{2} \mathrm{O}$; Ns), and the $P$ ab- $P$ pl relationship (fig. $2 \mathrm{~b}$ ) also remained unchanged.

Representative records of diaphragmatic, parasternal, and external intercostal activity during resting, control breathing and during in-phase vibration of the ninth interspace are shown in figure 3. No animal showed any inspiratory activity in the external intercostal muscle of the ninth interspace, and no animal had any consistent decrease or increase in diaphragmatic EMG activity during vibration. For the six animals studied, peak diaphragmatic activity in the control breaths thus averaged $99.4 \pm 1.0 \%$ of the peak activity recorded in the vibrated breaths (NS). Tidal volume (control: $272 \pm 45 \mathrm{~mL}$; vibration: $272 \pm$ $45 \mathrm{~mL}$; NS), $t$ I (control: $0.95 \pm 0.12 \mathrm{~s}$; vibration: $0.98 \pm$ 0.14 s; NS), $P$ di (control: $8.9 \pm 1.2 \mathrm{cmH}_{2} \mathrm{O}$; vibration: $8.9 \pm 1.2 \mathrm{cmH}_{2} \mathrm{O}$, NS), and the $P$ ab- $P$ pl relationship (fig. 2c) did not show any consistent alteration either.

\section{Discussion}

The current studies have confirmed the previous observation [13] that vibrating the ribcage during 

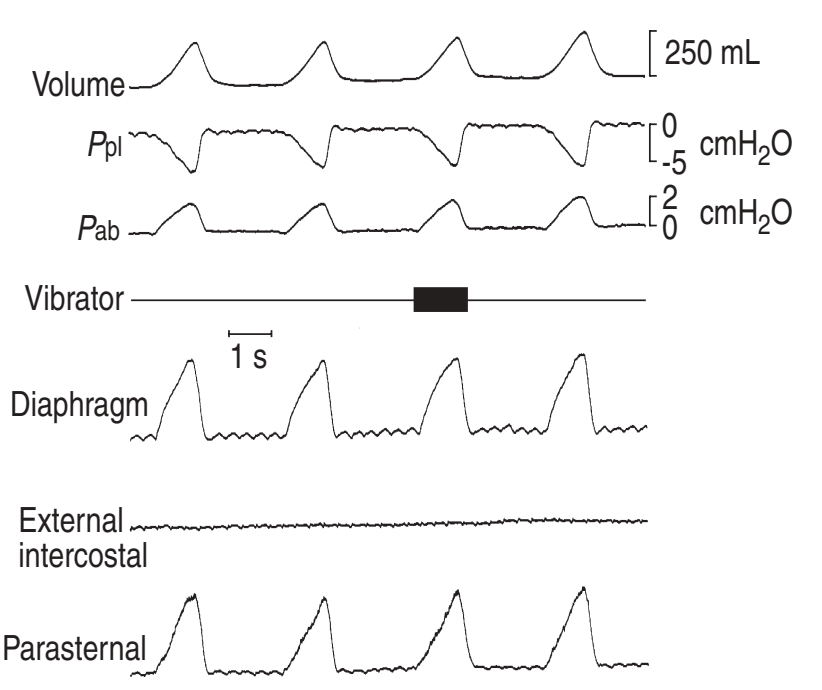

Fig. 3.-Electrical response of the diaphragm and external intercostal muscle (ninth interspace) to in-phase vibration of the ninth interspace in a representative animal. The trace of parasternal intercostal activity in the sixth interspace is also shown. The external intercostal remained silent throughout, including during vibration, and no alteration occurred in the pattern of diaphragm or parasternal intercostal activity. $P$ pl: pleural pressure; $P$ ab: abdominal pressure.

inspiration elicits an increased inspiratory activity in the external intercostal muscles without causing any alteration in the activity of the parasternal intercostals. As the authors have pointed out previously [13], this differential response to vibration is consistent with the earlier histological studies of Duron et al. [14] indicating that the external intercostals in the cat have a much greater density in muscle spindles than the parasternal intercostals. The finding that vibration in the animals in this study induced a four-fold increase in external intercostal activity in the third interspace, but only a two-fold increase in external intercostal activity in the sixth interspace, and did not elicit any activity in the ninth interspace is also consistent with the rostro-caudal gradient of spindle density in these muscles [14]. It must be pointed out, however, that in the dog, the neural drive to the external intercostal $\alpha$-motoneurones decreases markedly and continuously from the top to the base of the ribcage during inspiration, such that the muscles below the seventh interspace usually remain silent during breathing. This includes when breathing is stimulated by $\mathrm{CO}_{2}$-enriched gas mixtures or by elevated inspiratory mechanical loads [18]. A similar rostro-caudal gradient of neural drive to the external intercostals has been reported in the cat $[16,19]$, and indeed, no animal in this study had any inspiratory activity in the external intercostal of the ninth inter space during resting, unstimulated breathing. Presumably, the corresponding $\alpha$-motoneurones were well below their activation threshold, such that the increased spindle afferent activity produced by vibration was not sufficient to elicit efferent $\alpha$-motor activity to the muscle.

The main finding of these studies, however, is the observation that ribcage vibration did not induce any alteration in diaphragmatic EMG activity. Whether the vibrator was applied to the rostral, the middle, or the caudal portion of the ribcage, no change was seen either in $P$ di or in the relationship between $\Delta P$ ab and $\Delta P \mathrm{pl}$, thus indicating that the mechanical contribution of the diaphragm to inspiration was also unaltered [20]. Therefore, the conclusion must be drawn that ribcage vibration, while inducing a marked increase in afferent activity from external intercostal muscle spindles, has no inhibitory effect on the diaphragm. This excludes the possibility that the diaphragm would be responsible for the maintainance of tidal volume during this procedure [13]. The lack of diaphragmatic response to ribcage vibration, combined with the absence of alteration in $t \mathrm{I}$ or in parasternal intercostal inspiratory EMG activity, further indicates that the procedure has little or no supraspinal effect.

Earlier studies in cats and dogs by REMMERs [7] have shown a reflex, supraspinal reduction in diaphragmatic EMG activity during chest compression and during intercostal muscle stretch in the middle segments of the ribcage (T4-T7). In additional experiments, Remmers [8] and Decima et al. [21] have reported a similar inhibition of the diaphragm during electrical stimulation of group I and II afferent fibres in external intercostal nerves. Since this inhibitory response was also observed during high-frequency mechanical vibration of the ribs and was accentuated by the administration of succinylcholine, it was attributed to the stimulation of intercostal muscle spindles [7]. However, as Bolser et al. [22] have outlined subsequently, these electrical and mechanical stimuli were highly aspecific, and none of them, including rib vibration, could separate the effects of muscle spindle endings from those of Golgi tendon organs. Indeed, these vibrations were applied along the longitudinal axis of intercostal muscle fibres and elicited considerable changes in muscle length $(500-3,000 \mu \mathrm{m})$. Conversely, the vibrations in the animals in this study were applied perpendicular to the surface of the ribcage such that the changes in intercostal muscle length were only $90-100 \mu \mathrm{m}[13,15]$. Furthermore, BOLSER et al. [22], in agreement with the current findings, have found in decerebrate, unanaesthetized cats, that low-amplitude $(<100 \mu \mathrm{m})$ vibration of external and internal intercostal muscles, leading to exclusive stimulation of muscle spindles, has no effect on phrenic or medullary inspiratory activity. Inhibitory responses were observed only when the amplitude of vibration was increased above $700 \mu \mathrm{m}$ and produced concomitant stimulation of large numbers of intercostal tendon organs, thus indicating that the reflex inhibition of the diaphragm described by REMmers [7, 8] was due to tendon organ afferents, rather than muscle spindle afferents.

The question as to why ribcage vibration reduces dyspnoea in patients with COPD [1, 2] cannot be answered on the basis of the present study. In contrast to the animals in this study, such patients have an increased airflow resistance and a reduced dynamic pulmonary compliance. As a result, the resistive and elastic loads are greater than normal and the inspiratory muscles have to generate a greater reduction in $P$ pl to inflate the lung. Also, the emphysematous 
changes in the lung cause hyperinflation, which makes the inspiratory muscles operate at shorter than normal lengths and reduces their ability to lower intrathoracic pressure. Thus, COPD imposes a severe strain on the respiratory muscle pump, and patients accommodate it by increasing neural drive to both the ribcage inspiratory muscles [23] and the diaphragm [24, 25]. However, as in this study, vibrations in such patients are applied perpendicular to the surface of the ribcage. Consequently, the changes in length of the intercostal and superficial chest wall muscles are probably small, and since there is no reason to believe that mechanoreceptors in human and canine skeletal muscles differ in their sensitivity to vibration, it would be expected that the procedure in humans would also induce selective or predominant afferent activity from muscle spindles.

Therefore, in view of the current findings and of the observations of Bolser et al. [22], it would be unreasonable to maintain that the beneficial effect of ribcage vibration on dyspnoea results from a specific reduction in central respiratory drive.

\section{References}

1. Sibuya M, Yamada M, Kanamaru A, et al. Effect of chest wall vibration on dyspnea in patients with chronic respiratory disease. Am J Respir Crit Care Med 1994; 149: 1235-1240.

2. Christiano LM, Schwartzstein RM. Effects of chest wall vibration on dyspnea during hypercapnia and exercise in chronic obstructive pulmonary disease. $\mathrm{Am}$ J Respir Crit Care Med 1997; 155: 1552-1559.

3. Manning HL, Basner R, Ringler $\mathrm{J}$, et al. Effect of chest wall vibration on breathlessness in normal subjects. J Appl Physiol 1991; 71: 175-181.

4. Edo H, Kimura H, Nijima M, et al. Effects of chest wall vibration on breathlessness during hypercapnic ventilatory response. J Appl Physiol 1998; 84: 14871491.

5. Brown MC, Engberg I, Matthews PBC. The relative sensitivity to vibration of muscle receptors of the cat. J Physiol 1967; 192: 773-800.

6. Burke D, Hagbarth KE, Löfstedt L, Wallin BG. The responses of human muscle spindle endings to vibration of non-contracting muscles. J Physiol 1976; 261 : 673-693.

7. Remmers JE. Inhibition of inspiratory activity by intercostal muscle afferents. Respir Physiol 1970; 10: 358-383.

8. Remmers J. Extra-segmental reflexes derived from intercostal afferents: phrenic and laryngeal responses. J Physiol 1973; 233: 45-62.

9. Homma I, Eklund G, Hagbarth KG. Respiration in man affected by TVR contractions elicited in inspiratory and expiratory intercostal muscles. Respir Physiol 1978; 35: 335-348.

10. Killian KJ, Brucens DD, Campbell EJM. Effect of breathing patterns on the perceived magnitude of added loads to breathing. J Appl Physiol 1982; 52: 578-584.

11. Adams L, Lane R, Shea SA, Cockcroft A, Guz A. Breathlessness during different forms of ventilatory stimulation: a study of mechanisms in normal subjects and respiratory patients. Clin Sci 1985; 69: 663-672.

12. Freedman S, Lane R, Guz A. Breathlessness and respiratory mechanics during reflex or voluntary hyperventilation in patients with chronic airflow limitation. Clin Sci 1987; 73: 311-318.

13. Leduc D, Brunko E, De Troyer A. Response of the canine inspiratory intercostal muscles to chest wall vibration. Am J Respir Crit Care Med 2000; 161: 510516.

14. Duron B, Jung-Caillol MC, Marlot D. Myelinated nerve fiber supply and muscle spindles in the respiratory muscles of cat: quantitative study. Anat Embryol 1978; 152: 171-192.

15. Leduc D, Brunko E, De Troyer A. Response of the canine internal intercostal muscles to chest wall vibration. Am J Respir Crit Care Med 2001; 163: 4954.

16. De Troyer A, Legrand A. Inhomogeneous activation of the parasternal intercostals during breathing. $J$ Appl Physiol 1995; 79: 55-62.

17. Kirkwood PA, Sears TA, Stagg D, Westgaard RH. The spatial distribution of synchronisation of intercostal motoneurones in the cat. J Physiol 1982; 327: $137-155$.

18. Legrand A, De Troyer A. Spatial distribution of external and internal intercostal activity in dogs. J Physiol 1999; 518: 291-300.

19. Greer JJ, Martin TP. Distribution of muscle fiber types and EMG activity in cat intercostal muscle. J Appl Physiol 1990; 69: 1208-1211.

20. Macklem PT, Gross D, Grassino A, Roussos C. Partitioning of inspiratory pressure swings between diaphragm and intercostal/accessory muscles. $J$ Appl Physiol 1978; 44: 200-208.

21. Decima EE, von Euler C, Thoden U. Intercostal-tophrenic reflexes in the spinal cat. Acta Physiol Scand 1969; 75: 568-579.

22. Bolser DC, Lindsey BG, Shannon R. Medullary inspiratory activity: influence of intercostal tendon organs and muscle spindle endings. J Appl Physiol 1987; 62: 1046-1056.

23. Gandevia SC, Leeper JB, McKenzie DK, De Troyer A. Discharge frequencies of parasternal intercostal and scalene motor units during breathing in normal and COPD subjects. Am J Respir Crit Care Med 1996; 153: 622-628.

24. De Troyer A, Leeper JB, McKenzie DK, Gandevia SC. Neural drive to the diaphragm in patients with severe COPD. Am J Respir Crit Care Med 1997; 155: $1335-1340$.

25. Sinderby C, Beck J, Spahija J, Weinberg J, Grassino A. Voluntary activation of the human diaphragm in health and disease. J Appl Physiol 1998; 85: 21462158. 